\title{
Resonant spin-flavor conversion of supernova neutrinos: Dependence on presupernova models and future prospects
}

\author{
Shin'ichiro Andd* \\ Department of Physics, School of Science, the University of Tokyo, \\ 7-3-1 Hongo, Bunkyo-ku, Tokyo 113-0033, Japan \\ Katsuhiko Sato \\ Department of Physics, School of Science, the University of Tokyo, \\ 7-3-1 Hongo, Bunkyo-ku, Tokyo 113-0033, Japan and \\ Research Center for the Early Universe, School of Science, \\ the University of Tokyo, 7-3-1 Hongo, Bunkyo-ku, Tokyo 113-0033, Japan
}

(Dated: Received 22 March 2003; accepted 3 May 2003)

\begin{abstract}
We study the resonant spin-flavor (RSF) conversion of supernova neutrinos, which is induced by the interaction between the nonzero neutrino magnetic moment and the supernova magnetic fields, and its dependence on presupernova models. As the presupernova models, we adopt the latest ones by Woosley, Heger, and Weaver, and, further, models with both solar and zero metallicity are investigated. Since the $\left(1-2 Y_{e}\right)$ profile of the new presupernova models, which is responsible for the RSF conversion, suddenly drops at the resonance region, the completely adiabatic RSF conversion is not realized, even if $\mu_{\nu} B_{0}=\left(10^{-12} \mu_{B}\right)\left(10^{10} \mathrm{G}\right)$, where $B_{0}$ is the strength of the magnetic field at the surface of the iron core. In particular for the model with zero metallicity, the conversion is highly nonadiabatic in the high energy region, reflecting the $\left(1-2 Y_{e}\right)$ profile of the model. In calculating the flavor conversion, we find that the shock wave propagation, which changes density profiles drastically, is a much more severe problem than it is for the pure Mikheyev-Smirnov-Wolfenstein (MSW) conversion case. This is because the RSF effect occurs at a far deeper region than the MSW effect. To avoid the uncertainty concerning the shock propagation, we restrict our discussion to $0.5 \mathrm{~s}$ after the core bounce (and for more conservative discussion, $0.25 \mathrm{~s}$ ), during which the shock wave is not expected to affect the RSF region. We also evaluate the energy spectrum at the SuperKamiokande detector for various models using the calculated conversion probabilities, and find that it is very difficult to obtain useful information on the supernova metallicities and magnetic fields or on the neutrino magnetic moment from the supernova neutrino observation. Future prospects are also discussed.
\end{abstract}

PACS numbers: 95.85.Ry, 13.40.Em, 14.60.Pq, 97.60.Bw

\section{INTRODUCTION}

A core-collapse supernova explosion is one of the most spectacular events in astrophysics; $99 \%$ of its gravitational binding energy is released as neutrinos, and only $1 \%$ as the kinetic energy of the explosion. Therefore, neutrinos play an essential role in supernovae, and their detection by ground-based large water Čerenkov detectors, such as Super-Kamiokande (SK) and Sudbury Neutrino Observatory (SNO), would provide valuable information not only on supernova physics but also on the nature of neutrinos. What we can learn from the next galactic supernova has been considered in many articles (for a review, see Ref. [1]). For example, we can constrain the properties of neutrino oscillations, such as the mixing angle between the first and third mass eigenstates $\left(\theta_{13}\right)$, and the mass hierarchy [normal $\left(m_{1} \ll m_{3}\right)$ or inverted $\left.\left(m_{1} \gg m_{3}\right)\right][2,[3]$.

In addition to the nonzero neutrino masses and mixing angles, the nonzero magnetic moment is of a differ-

\footnotetext{
*Email address: ando@utap.phys.s.u-tokyo.ac.jp
}

ent nature for neutrinos beyond the standard model of particle physics, and has attracted a great deal of attention from many theoretical and experimental physicists. If neutrinos have a nonzero magnetic moment, it leads to precession between left- and right-handed neutrinos in sufficiently strong magnetic fields [4, 5]. In general, nondiagonal elements of the magnetic moment matrix are possible and neutrinos can be changed into different flavors and chiralities [6, 7]. Furthermore, with the additional effect of coherent forward scattering by matter, neutrinos can be resonantly converted into those with different chiralities $8,0,10$ by a mechanism similar to the well-known Mikheyev-Smirnov-Wolfenstein (MSW) effect [11, 12, 13]. This resonant spin-flavor (RSF) conversion induced by the neutrino magnetic moment in strong magnetic fields was first introduced to solve the solar neutrino problem, and actually gave the best fit solution before the KamLAND result 14]. However, the recent KamLAND experiment 15] has shown that the large mixing angle MSW solution is the most favorable one; the RSF mechanism is suppressed at the subdominant level. From the KamLAND negative results for the solar antineutrino search, an upper bound on the neutrino magnetic moment is obtained, $\mu_{\nu} \lesssim 1 \times 10^{-12} \mu_{B}$, 
where $\mu_{B}$ is the Bohr magneton [16]. This upper bound is comparable to the most stringent limit from the stellar cooling argument, $\mu_{\nu} \lesssim(1-4) \times 10^{-12} \mu_{B}$ [17].

Although the RSF mechanism does not work at a dominant level in the Sun, it may occur efficiently in a denser environment with stronger magnetic field, which is actually expected in the case of core-collapse supernovae. The RSF conversion mechanism in supernovae has been investigated by many authors [8, 9, 10, 18, 19, 20, 21, 22, 23, 24, 25, 26]. Among them, Ando and Sato 26] studied the RSF effect using a three-flavor formulation with the latest oscillation parameters, and pointed out that the combination of the MSW and RSF effects makes the crossing scheme very interesting to investigate. They showed that until $0.5 \mathrm{~s}$ after the core bounce the RSF-induced $\bar{\nu}_{e} \leftrightarrow \nu_{\tau}$ transition occurs efficiently, when $\mu_{\nu} \gtrsim 10^{-12} \mu_{B}\left(B_{0} / 5 \times 10^{9} \mathrm{G}\right)^{-1}$, where $B_{0}$ is the strength of the magnetic field at the surface of the iron core.

The effective matter potential for the RSF conversion is given in a form proportional to the value of $\left(1-2 Y_{e}\right)$, where $Y_{e}$ is the electron number fraction per nucleon. Thus, the deviation of the value of $Y_{e}$ from 0.5 in the stellar envelope is quite important, and this value is strongly dependent on the isotopic composition. Since this deviation is determined by rarely existent nuclei, an accurate estimate of this deviation is quite difficult. Therefore, the astrophysical uncertainty in $\left(1-2 Y_{e}\right)$ should be discussed. This point was first investigated by Totani and Sato [23]. However, their treatment was based on a twoflavor formulation with the large uncertainties concerning the mixing parameters of those days. Therefore, we need definitive investigations using the three-flavor formulation with the latest presupernova models as well as the recently determined neutrino parameters.

In this paper, we study the RSF conversion mechanism using the three-flavor formulation with the latest neutrino mixing parameters. In particular, we investigate the dependence on presupernova models; we use the latest $15 M_{\odot}$ model by Woosley et al. 27], and compare the results with those obtained with the previous progenitor model by Woosley and Weaver [28], which was also adopted in the calculations of Ando and Sato [26]. It is also expected that the value of $\left(1-2 Y_{e}\right)$ strongly depends on the stellar metallicity, and hence we use the $15 M_{\odot}$ model with two different metallicities, solar and zero metallicity, and the metallicity effect is investigated.

Throughout this paper, we adopt the realistic neutrino mixing parameters inferred from the recent experimental results: for the atmospheric neutrino parameters, $\Delta m_{13}^{2}=2.8 \times 10^{-3} \mathrm{eV}^{2}, \sin ^{2} 2 \theta_{23}=1.0$, and for the solar neutrino parameters, $\Delta m_{12}^{2}=5.0 \times 10^{-5} \mathrm{eV}^{2}, \tan ^{2} \theta_{12}=$ 0.42 . As for the still uncertain parameter $\theta_{13}$, we assume $\sin ^{2} 2 \theta_{13}=10^{-6}, 1$ and we also assume a normal

\footnotetext{
1 As already shown in Ref. [26], the dependence of the detected event on the parameter $\theta_{13}$ is very weak, and even if we assume
}

mass hierarchy. For the elements of the neutrino magnetic moment matrix $\mu_{i j}$, where $i$ and $j$ denote the flavor eigenstates of the neutrinos, i.e., $e, \mu$, and $\tau$, we assume that all the values of $\mu_{i j}$ are near the current upper limit, or $\mu_{i j}=10^{-12} \mu_{B}$.

The remainder of this paper is organized as follows. In Sec. II we give the formulation used in our calculation, which includes all three-flavor neutrinos and antineutrinos, and from the formulation a level crossing diagram, which enables us to understand the conversion scheme intuitively, is introduced. In Sec. III the presupernova model we adopt in our calculations is illustrated, and the results of numerical calculations are shown in Sec. [IV] Finally, detailed discussions of the model uncertainties and of whether we can obtain, at present or in the future, useful information on physical and astrophysical quantities are presented in Sec. V

\section{FORMULATION AND LEVEL CROSSING SCHEME}

\section{A. Interaction with matter and magnetic fields}

The interaction of the magnetic moment of neutrinos and magnetic fields is described by

$$
\left\langle\left(\nu_{i}\right)_{R}\left|H_{\mathrm{int}}\right|\left(\nu_{j}\right)_{L}\right\rangle=\mu_{i j} B_{\perp}
$$

where $B_{\perp}$ is the magnetic field transverse to the direction of propagation, and $(\nu)_{R}$ and $(\nu)_{L}$ are the rightand left-handed neutrinos, respectively. If neutrinos are Dirac particles, right-handed neutrinos and left-handed antineutrinos are undetectable (sterile neutrinos), since they do not interact with matter. On the other hand, if neutrinos are Majorana particles, $\nu_{R}$ 's are identical to antiparticles of $\nu_{L}$ 's and interact with matter. In this paper, we assume that neutrinos are Majorana particles. The diagonal magnetic moments are forbidden for Majorana neutrinos, and therefore only conversion between different flavors is possible, e.g., $\left(\bar{\nu}_{e}\right)_{R} \leftrightarrow\left(\nu_{\mu, \tau}\right)_{L}$.

Coherent forward scattering with matter induces an effective potential for neutrinos, which is calculated using weak interaction theory. The effective potential due to scattering with electrons is given by

$$
V_{ \pm \pm}= \pm \sqrt{2} G_{F}\left( \pm \frac{1}{2}+2 \sin ^{2} \theta_{W}\right) n_{e}
$$

where $n_{e}$ is the electron number density, $G_{F}$ is the Fermi coupling constant, and $\theta_{W}$ is the Weinberg angle. The \pm sign in front refers to $\nu(+)$ and $\bar{\nu}(-)$ and that in the parentheses to $\nu_{e}(+)$ and $\nu_{\mu, \tau}(-)$. The difference between $e$ and $\mu, \tau$ neutrinos comes from the existence of charged-current interaction. The subscript \pm \pm of $V$

a large value of $\theta_{13}$, the results do not change. 
refers to the first and the second \pm sign. The ordinary MSW effect between $\nu_{e}$ and $\nu_{\mu, \tau}$ is caused by the potential difference $V_{e}-V_{\mu, \tau}=V_{++}-V_{+-}=\sqrt{2} G_{F} n_{e}$. To include the RSF effect, which causes conversion between neutrinos and antineutrinos, we should take into account the neutral-current scattering by nucleons:

$$
V=\sqrt{2} G_{F}\left(\frac{1}{2}-2 \sin ^{2} \theta_{W}\right) n_{p}-\sqrt{2} G_{F} \frac{1}{2} n_{n},
$$

where $n_{p}, n_{n}$ are the proton and neutron number density, respectively. For neutrinos we add $+V$ to the potential and for antineutrinos $-V$. Therefore, the RSF conversion between $\bar{\nu}_{e}$ and $\nu_{\mu, \tau}$ obeys the potential difference

$$
\begin{aligned}
\Delta V & \equiv V_{\bar{e}}-V_{\mu, \tau} \\
& =\left(V_{-+}-V\right)-\left(V_{+-}+V\right) \\
& =\sqrt{2} G_{F} \frac{\rho}{m_{N}}\left(1-2 Y_{e}\right),
\end{aligned}
$$

where $\rho$ is the density, $m_{N}$ is the nucleon mass, and $Y_{e}=n_{e} /\left(n_{e}+n_{n}\right)$ is the number of electrons per baryon. [When we obtained Eq. (4), we assumed charge neutrality $n_{e}=n_{p}$.]

\section{B. Three-flavor formulation}

The time evolution of the flavor mixing, which includes all three-flavor neutrinos and antineutrinos, is described by the Schrödinger equation

$$
i \frac{d}{d r}\left(\begin{array}{c}
\nu \\
\bar{\nu}
\end{array}\right)=\left(\begin{array}{cc}
H_{0} & B_{\perp} M \\
-B_{\perp} M & \bar{H}_{0}
\end{array}\right)\left(\begin{array}{c}
\nu \\
\bar{\nu}
\end{array}\right),
$$

where

$$
\begin{aligned}
& \nu=\left(\begin{array}{c}
\nu_{e} \\
\nu_{\mu} \\
\nu_{\tau}
\end{array}\right), \quad \bar{\nu}=\left(\begin{array}{c}
\bar{\nu}_{e} \\
\bar{\nu}_{\mu} \\
\bar{\nu}_{\tau}
\end{array}\right) \\
& H_{0}=\frac{1}{2 E_{\nu}} U\left(\begin{array}{ccc}
0 & 0 & 0 \\
0 & \Delta m_{12}^{2} & 0 \\
0 & 0 & \Delta m_{13}^{2}
\end{array}\right) U^{\dagger}+\left(\begin{array}{ccc}
V_{++}+V & 0 & 0 \\
0 & V_{+-}+V & 0 \\
0 & 0 & V_{+-}+V
\end{array}\right) \\
& \bar{H}_{0}=\frac{1}{2 E_{\nu}} U\left(\begin{array}{ccc}
0 & 0 & 0 \\
0 & \Delta m_{12}^{2} & 0 \\
0 & 0 & \Delta m_{13}^{2}
\end{array}\right) U^{\dagger}+\left(\begin{array}{ccc}
V_{-+}-V & 0 & 0 \\
0 & V_{--}-V & 0 \\
0 & 0 & V_{--}-V
\end{array}\right) \\
& U=\left(\begin{array}{ccc}
U_{e 1} & U_{e 2} & U_{e 3} \\
U_{\mu 1} & U_{\mu 2} & U_{\mu 3} \\
U_{\tau 1} & U_{\tau 2} & U_{\tau 3}
\end{array}\right)=\left(\begin{array}{ccc}
c_{12} c_{13} & s_{12} c_{13} & s_{13} \\
-s_{12} c_{23}-c_{12} s_{23} s_{13} & c_{12} c_{23}-s_{12} s_{23} s_{13} & s_{23} c_{13} \\
s_{12} s_{23}-c_{12} c_{23} s_{13} & -c_{12} s_{23}-s_{12} c_{23} s_{13} & c_{23} c_{13}
\end{array}\right) \text {, } \\
& M=\left(\begin{array}{ccc}
0 & \mu_{e \mu} & \mu_{e \tau} \\
-\mu_{e \mu} & 0 & \mu_{\mu \tau} \\
-\mu_{e \tau} & -\mu_{\mu \tau} & 0
\end{array}\right)
\end{aligned}
$$

and $c_{i j}=\cos \theta_{i j}, s_{i j}=\sin \theta_{i j}$. [We assume the $C P$ phase $\delta=0$ in Eq. (9) for simplicity.]

Resonant flavor conversion occurs when two diagonal elements of the matrix in Eq. (5) have the same value. There are five such resonance points, which are for $\nu_{e} \leftrightarrow$ $\nu_{\mu}$ (MSW-L), $\nu_{e} \leftrightarrow \nu_{\tau}$ (MSW-H), $\bar{\nu}_{e} \leftrightarrow \nu_{\mu}$ (RSF-L), $\bar{\nu}_{e} \leftrightarrow \nu_{\tau}$ (RSF-H), and $\bar{\nu}_{\mu} \leftrightarrow \nu_{\tau}$ conversions. The suffixes "-L" and "-H" attached to "MSW" and "RSF" indicate whether the density at the resonance points is lower or higher. Hereafter, we neglect the $\bar{\nu}_{\mu} \leftrightarrow \nu_{\tau}$ conversion, since it is always nonadiabatic and including it further complicates the discussion.
Figure 1 shows the level crossing diagram, which we introduced in our previous paper [26], to understand the flavor conversions described by Eq. (5) intuitively. The figure clearly includes not only the ordinary MSW resonances but also the RSF effects, and it is expected that the combined effect of MSW and RSF makes this scheme very interesting to investigate. For instance, when MSW$\mathrm{L}$ and $\mathrm{RSF}-\mathrm{H}$ are adiabatic and the others are nonadiabatic (this case is actually expected if $\theta_{13}$ is small and the magnetic field is sufficiently strong), conversions such as $\nu_{e} \rightarrow \nu_{2}, \nu_{\mu}^{\prime} \rightarrow \nu_{1}, \nu_{\tau}^{\prime} \rightarrow \bar{\nu}_{1}, \bar{\nu}_{e} \rightarrow \nu_{3}, \bar{\nu}_{\mu}^{\prime} \rightarrow \bar{\nu}_{2}$, and $\bar{\nu}_{\tau}^{\prime} \rightarrow \bar{\nu}_{3}$ occur. We can easily predict this sort of conversion scheme from Fig. 1] when all the resonances are 


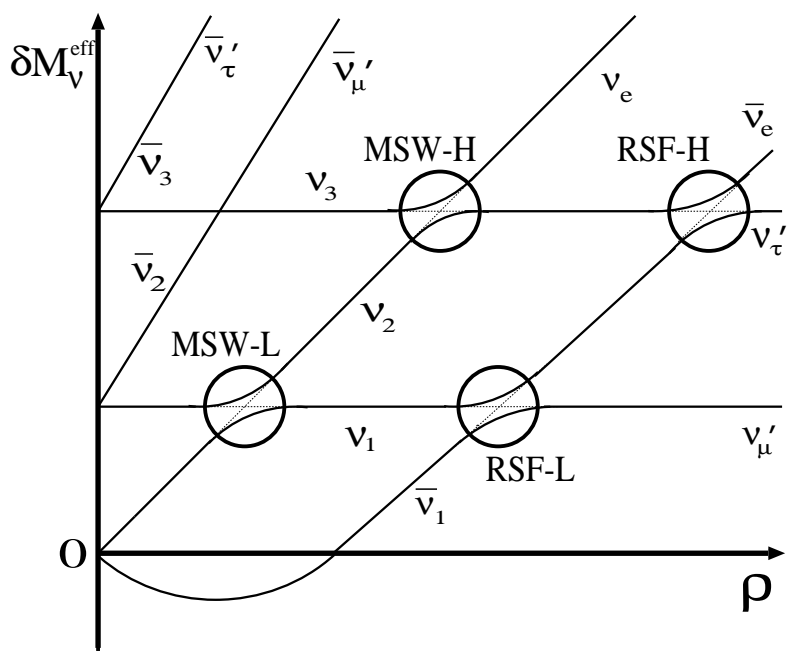

FIG. 1: Schematic illustration of level crossings, where $\nu_{1,2,3}$ and $\bar{\nu}_{1,2,3}$ represent the mass eigenstates of neutrinos and antineutrinos in matter, respectively, and $\nu_{\mu, \tau}^{\prime}$ and $\bar{\nu}_{\mu, \tau}^{\prime}$ the mass eigenstates at production, which are superpositions of $\nu_{\mu}$ and $\nu_{\tau}$ or $\bar{\nu}_{\mu}$ and $\bar{\nu}_{\tau}$. There are four resonance points, MSWL, MSW-H, RSF-L, and RSF-H. Adiabatic conversion means that the neutrinos trace the solid curve at each resonance point (i.e., the mass eigenstate does not flip), while nonadiabatic conversion is shown by the dotted line.

either completely adiabatic or completely nonadiabatic; for the intermediate cases we have no choice but to trust numerical calculations.

\section{PRESUPERNOVA MODELS}

\section{A. Density and $Y_{e}$ profiles}

In this subsection, we discuss the several presupernova models that we adopt in our transition calculations. In a previous paper [26], we used only the presupernova model of Woosley and Weaver [28], which is for $15 M_{\odot}$ and solar metallicity (hereafter W95S, where "S" denotes solar metallicity). However, we should investigate the dependence on adopted presupernova models with various metallicities. This is because the RSF conversion is very sensitive to the deviation of $Y_{e}$ from 0.5 [see Eq. (4)], which strongly depends on the metallicities as well as on the weak interaction rates adopted in the simulation of stellar evolution.

In this study, we adopt the latest presupernova models by Woosley et al. 27] with both solar and zero metallicities: W02S and W02Z ("Z" denotes zero metallicity). Figures 2 and 3 show density profiles (upper panel) or $\rho Y_{e}$, which is responsible for MSW, and $\left|\rho\left(1-2 Y_{e}\right)\right|$ for RSF, and the composition of each element (lower panel), for the W02S and W02Z models, respectively. In the upper panels of both figures, we also show $\Delta_{12} \equiv m_{N} \Delta m_{12}^{2} \cos 2 \theta_{12} / 2 \sqrt{2} G_{F} E_{\nu}$ and $\Delta_{13} \equiv$

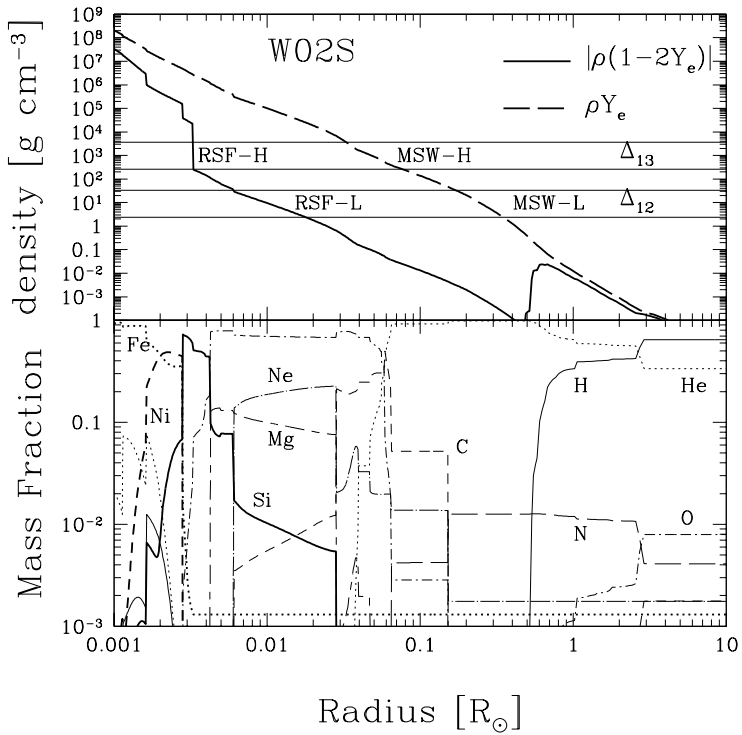

FIG. 2: Presupernova profiles (W02S) used in our calculations taken from Ref. [27]. Upper panel: The density and $Y_{e}$ combination that is responsible for the RSF conversions $\left[\left|\rho\left(1-2 Y_{e}\right)\right|\right.$, solid curve], and that for the MSW conversions ( $\rho Y_{e}$, dashed curve). Two horizontal bands represent $\Delta_{12}$ and $\Delta_{13}$ (these definitions are given in the text); at the intersections between them and the profile curves, the RSF and MSW conversions occur. Lower panel: The mass fraction of the various elements.

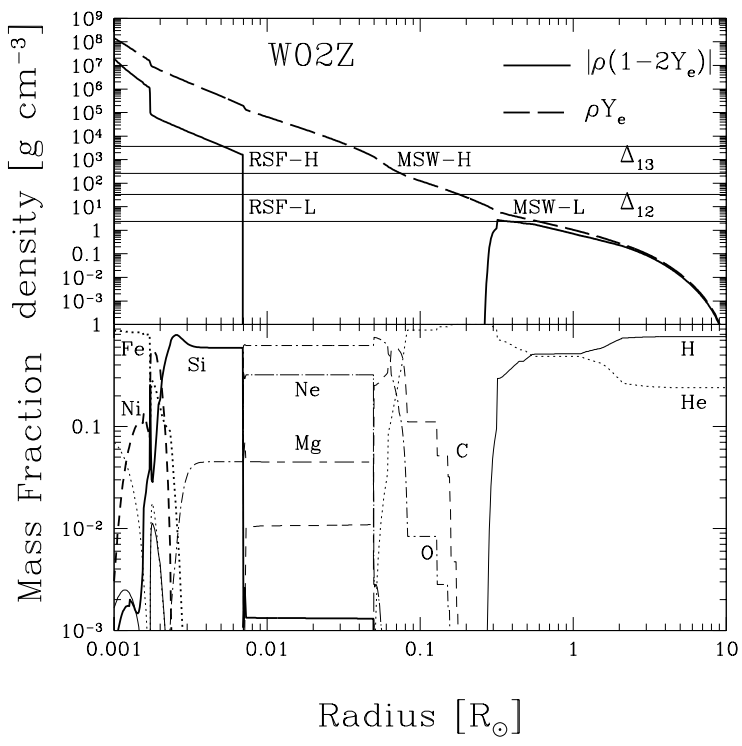

FIG. 3: The same as Fig. 2 but for the W02Z model. 
$m_{N} \Delta m_{13}^{2} \cos 2 \theta_{13} / 2 \sqrt{2} G_{F} E_{\nu}$ as two horizontal bands (the bandwidth comes from the energy range 5-70 MeV). At intersections between $\Delta_{12}, \Delta_{13}$ and $\rho\left(1-2 Y_{e}\right), \rho Y_{e}$, the $\mathrm{RSF}$ and MSW conversions take place.

For the W02S model, the region where RSF-H occurs is the silicon burning shell, in which $\left(1-2 Y_{e}\right)$ suddenly drops, in contrast with RSF-L, where $\rho\left(1-2 Y_{e}\right)$ gradually changes. On the other hand, for the W02Z model, $\left(1-2 Y_{e}\right)$ suddenly becomes exactly zero at the boundary between $\mathrm{Si}+\mathrm{O}$ and $\mathrm{O}+\mathrm{Ne}+\mathrm{Mg}$ layers, and this tendency continues to the He layer because of the lack of heavy nuclei, which cause the deviation of $Y_{e}$ from 0.5 . In consequence, both the RSF-H and RSF-L conversions are expected to be highly nonadiabatic, as discussed in more detail in Sec. IV] Note that the $\rho Y_{e}$ profiles of the two different models agree well with each other, which clearly indicates that the ordinary MSW conversions are not noticeably affected by the value of $Y_{e}$.

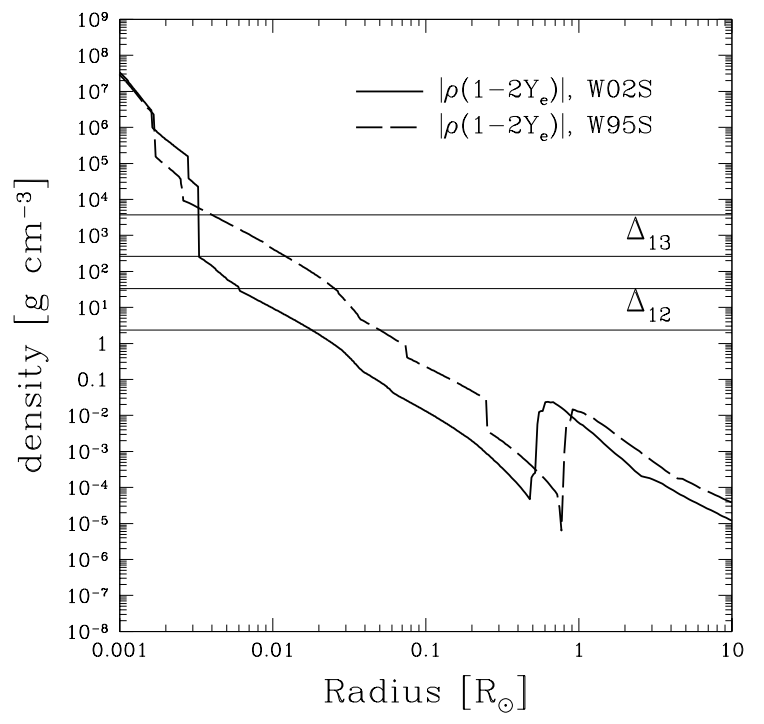

FIG. 4: The $\left|\rho\left(1-2 Y_{e}\right)\right|$ profiles, which are responsible for the RSF conversions, for the W02S (solid curve) and W95S (dashed curve) models.

In Fig. 4 we compare the $\left|\rho\left(1-2 Y_{e}\right)\right|$ profile of the W02S model with that of the W95S model, which was adopted in previous publications including Ref. [26]. The difference comes from the included weak interaction rates for nuclei. In the latest model W02S, a recent shell model is included in the calculations and results in substantial revisions to the older data set in the W95S model (see Ref. 29] for a detailed discussion). As a result, the value of $\left(1-2 Y_{e}\right)$ decreases by a few orders of magnitude between the $\mathrm{He}$ and $\mathrm{Si}+\mathrm{O}$ shells. Particularly with the new model W02S, there is a sudden drop of $\rho\left(1-2 Y_{e}\right)$ when the RSF-H conversion occurs, in contrast with the gradual decrease in the case of the W95S model. Since the adiabaticity of the resonance is reciprocal to the gra- dient of the logarithmic value of $\rho\left(1-2 Y_{e}\right)$, the RSF conversion is expected to be less efficient than that with W95S given in Ref. [26].

Although we use static progenitor models in calculating the flavor transition, in fact the density profile changes drastically during a neutrino burst ( $\sim 10 \mathrm{~s})$ owing to shock wave propagation, and we should use the time-dependent profiles [30]. Unfortunately, however, supernova explosion mechanisms are still controversial, and there is no reliable model that precisely describes the time-dependent density and $Y_{e}$ profiles. Further, there is also a large uncertainty concerning the magnetic field structure which is affected by the shock wave propagation. Therefore, from this point on, we confine our discussion to $0.5 \mathrm{~s}$ after core bounce, since in that case using the static presupernova and magnetic field models is considered to be a good approximation [26]. This is based on the numerical calculation by Takahashi et al. [31], the only authors having succeeded in shock propagation to the outer envelope. Although we cannot trust the details of their result without any doubt, the choice of the time scale during which the shock effect can be neglected is expected to be reasonable. In addition, for more conservative discussions, we also give calculations during first $0.25 \mathrm{~s}$.

\section{B. Magnetic fields}

We assume that the global structure of the magnetic field is a dipole moment and the field strength is normalized at the surface of the iron core with the values of $10^{8}$ and $10^{10} \mathrm{G}$. The reason for this normalization is as follows. The magnetic fields should be normalized by fields that are static and exist before the core collapse, because those of a nascent neutron star can hardly affect the far outer region, where the RSF conversions take place, within the short time scale of a neutrino burst. As discussed in the previous subsection, since the shock wave does not affect the resonance region at $\lesssim 0.5 \mathrm{~s}$ after bounce, it is also expected that the magnetic field structure and strength at the resonance points are not seriously changed at that time. The strength of such magnetic fields above the surface of the iron core may be inferred from observations of the surface of white dwarfs, since both are sustained against gravitational collapse by the degenerate pressure of electrons. Observations of the magnetic fields in white dwarfs show that the strength spreads in a wide range of $10^{7}-10^{9} \mathrm{G}[32$. Considering the possibility of the decay of magnetic fields in white dwarfs, it is not unnatural to consider magnetic fields up to $10^{10} \mathrm{G}$ at the surface of the iron core. Then, in Eq. (5), $B_{\perp}=B_{0}\left(r_{0} / r\right)^{3} \sin \Theta$, where $B_{0}$ is the strength of the magnetic field at the equator on the iron core surface, $r_{0}$ the radius of the iron core, and $\Theta$ the angle between the pole of the magnetic dipole and the direction of neutrino propagation. Hereafter, we assume $\sin \Theta=1$. 


\section{RESULTS}

\section{A. Conversion probabilities}

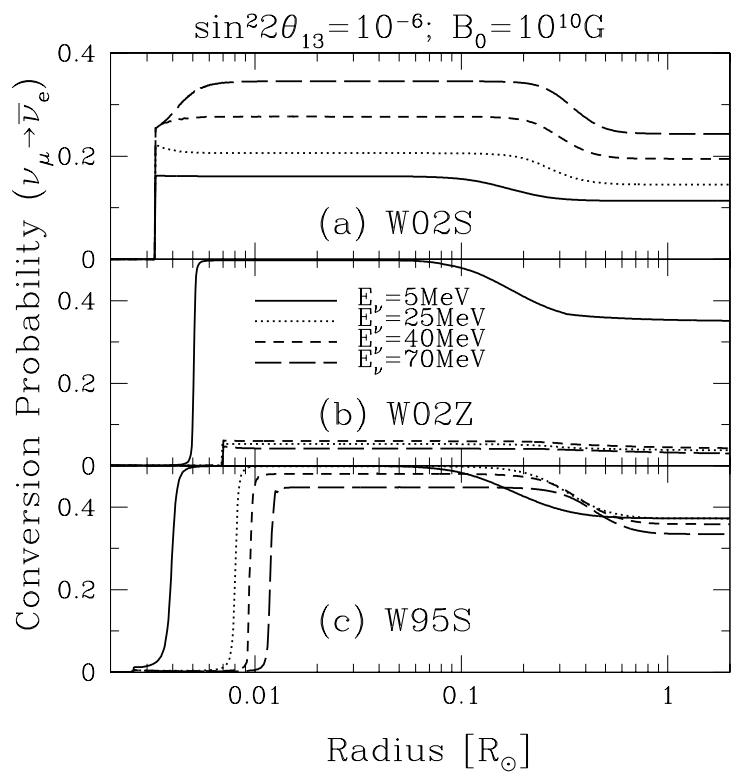

FIG. 5: Conversion probability $P\left(\nu_{\mu} \rightarrow \bar{\nu}_{e}\right)$ as a function of radius for $B_{0}=10^{10} \mathrm{G}$. The probabilities calulated with the W02S (a), W02Z (b), and W95S (c) models are plotted.

We calculated Eq. (5) numerically with the adopted models given in Sec. IIII and obtained the conversion probabilities for each flavor. Among them, we show in Fig. [5 those of the $\nu_{\mu} \rightarrow \bar{\nu}_{e}$ transition for various presupernova models in the case of $B_{0}=10^{10} \mathrm{G}$. (This conversion channel is essential in order to discuss the efficiency of the RSF effects.)

For the W02S model, Fig. [5 (a) shows that the conversion between $\nu_{\mu, \tau}$ and $\bar{\nu}_{e}$ occurs at a radius independent of energy, and becomes adiabatic as the energy increases; this character reflects the $\rho\left(1-2 Y_{e}\right)$ profile given in Fig. 2 which shows a sudden drop in the RSF-H region.

The behavior changes dramatically when the metallicity is zero, or for the W02Z model. In this case, as shown in Fig. 5(b), the transition is completely adiabatic at low energy; however, once the energy is increased beyond some critical value, the conversion abruptly becomes almost completely nonadiabatic. This tendency also reflects the characteristic profile in Fig. 3

The flavor transition occurs most efficiently in the case of the W95S model as shown in Fig. 5. (c), which indicates the most moderate profile at each resonance point (see

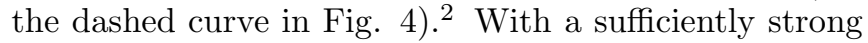

\footnotetext{
${ }^{2}$ Actually, the results for the W95S model are taken from previous
}

magnetic field $B_{0}=10^{10} \mathrm{G}$, the $\nu_{\mu} \rightarrow \bar{\nu}_{e}$ transition is almost completely adiabatic over the entire energy range.

For all these three models, it appears that the RSF-L conversion does not play any role. This is because for the W02S and W95S models RSF-L occurs farther out than RSF-H, where the magnetic field strength is not large enough to induce adiabatic conversions. On the other hand, for the W02Z model, the RSF-L occurs in the same place as RSF-H; the magnetic field strength is also the same for these two resonances. In this case, however, the very abrupt drop of the $\rho\left(1-2 Y_{e}\right)$ profile at RSF-L strongly suppresses efficient flavor conversions.

At the end of this subsection, we focus on the decrease of the conversion probability at $\gtrsim 0.1 R_{\odot}$ which can be seen in Fig. 5 This does not indicate any resonances but is merely an effect of flavor mixings. At such a deep region in the supernova envelope as $\lesssim 0.1 R_{\odot}$, the $\bar{\nu}_{e}$ 's propagate like the mass eigenstates owing to the large matter potential; the $\bar{\nu}_{e}$ 's do not mix with the other flavor antineutrinos in this region. As they propagate to a radius that is larger than $\sim 0.1 R_{\odot}$, the matter potential becomes smaller; at this stage the $\bar{\nu}_{e}$ 's are not the mass eigenstates at all, and mixing with the other flavor antineutrinos occurs. This effect induces the decrease at $\gtrsim 0.1 R_{\odot}$ seen in Fig. [5]

\section{B. Energy spectrum at the Super-Kamiokande detector}

With the conversion probabilities given in the previous subsection and the original neutrino spectrum emitted from the supernova core, we can calculate the flux of each flavor neutrino. From this point on, we assume that the distance to the supernova is $10 \mathrm{kpc}$. As the original neutrino spectrum, we adopt the results by two groups: the Lawrence Livermore group [33] and Thompson et al. 34]. The Livermore spectrum [33] resulted from a calculation with $20 M_{\odot}$ progenitor models, and we label it LL20. Recently, that calculation has been criticized, since it lacks relevant neutrino processes such as neutrino bremsstrahlung and neutrino-nucleon scattering with nucleon recoils, which were not recognized to be important at date of calculation. However, since there are no other successful simulations of a supernova explosion, it is premature to conclude that their result is no longer reliable and we adopt their results. On the other hand, Thompson et al. 34 calculated for three different mass progenitors $11 M_{\odot}, 15 M_{\odot}$, and $20 M_{\odot}$, and we label the models TBP11, TBP15, and TBP20, respectively. Although they did not succeed in simulating the explosion and their data end at $0.25 \mathrm{~s}$ after the core bounce,

calculations (Ref. 26]) with a slightly different mixing parameter, $\tan ^{2} \theta_{12}=0.34$ instead of 0.42 in the new calculations. However, note that the difference due to the parameter choice is quite small, as shown in the next subsection. 
they included all the relevant neutrino processes in their calculations.

Using the flux of each flavor neutrino on the Earth and cross sections of the relevant neutrino interactions at SK, we can calculate the expected event numbers from future galactic supernova neutrino bursts. SK is a water Cerenkov detector with 32 ktons of pure water, based at Kamioka in Japan. The relevant interactions of neutrinos with water are

$$
\begin{aligned}
\bar{\nu}_{e}+p & \rightarrow e^{+}+n(\mathrm{CC}), \\
\nu_{e}+e^{-} & \rightarrow \nu_{e}+e^{-}(\mathrm{CC} \text { and } \mathrm{NC}), \\
\bar{\nu}_{e}+e^{-} & \rightarrow \bar{\nu}_{e}+e^{-}(\mathrm{CC} \text { and } \mathrm{NC}), \\
\nu_{x}+e^{-} & \rightarrow \nu_{x}+e^{-}(\mathrm{NC}), \\
\nu_{e}+\mathrm{O} & \rightarrow e^{-}+\mathrm{F}(\mathrm{CC}), \\
\bar{\nu}_{e}+\mathrm{O} & \rightarrow e^{+}+\mathrm{N}(\mathrm{CC}),
\end{aligned}
$$

where $\mathrm{CC}$ and $\mathrm{NC}$ stand for charged- and neutral-current interactions, respectively. SK has restarted observation with lower performance, finishing the repair of the unfortunate accident which occurred on 12th November 2001. The effect of the accident on its performance is expected not to be serious for supernova neutrinos, because the fiducial volume does not change, and the threshold energy change (from $5 \mathrm{MeV}$ to about 7-8 MeV) influences the event number very little. Although the energy resolution will become about $\sqrt{2}$ times worse, it does not matter for our considerations. In the calculations, we used the energy threshold and the energy resolution after the accident, or in the so-called SK-II phase. For the cross section of the most relevant interaction Eq. (11), we referred to the recent result by Strumia and Vissani 35], and for the other processes we adopted the same ones as in Ref. [26]. As a result, the expected events decreased by $\sim 10 \%$ in contrast with the previous calculation in Ref. [26] under the same conditions but for the $\bar{\nu}_{e} p$ reaction cross section.

The expected event number per unit energy range is shown in Figs. [6] and 7] Figure [6] shows the energy spectrum of electrons (positrons) for the first $0.5 \mathrm{~s}$ with the LL20 model, which was obtained by conversion calculation with the W02S and W95S presupernova models. When the magnetic field is not strong, $B_{0}=10^{8} \mathrm{G}$, the RSF conversions are absent, leading to an energy spectrum independent of the presupernova model. (The slight difference between the two models comes from the difference in the adopted mixing angle, i.e., $\tan ^{2} \theta_{12}=0.42$ for W02S and 0.34 for W95S.) On the other hand, when the magnetic field is sufficiently strong, $B_{0}=10^{10} \mathrm{G}$, the energy spectrum is very sensitive to the adopted presupernova model, reflecting the results of conversion probabilities, Fig. 5 Since the flavor conversions are not as efficient in the case of the calculations with the W02S model as those with the W95S model, the expected energy spectrum with the W02S model is not as hard as that with the W95S model.

Figure 7 shows the energy spectrum at SK, obtained using the W02S and W02Z models with $B_{0}=10^{8}, 10^{10}$

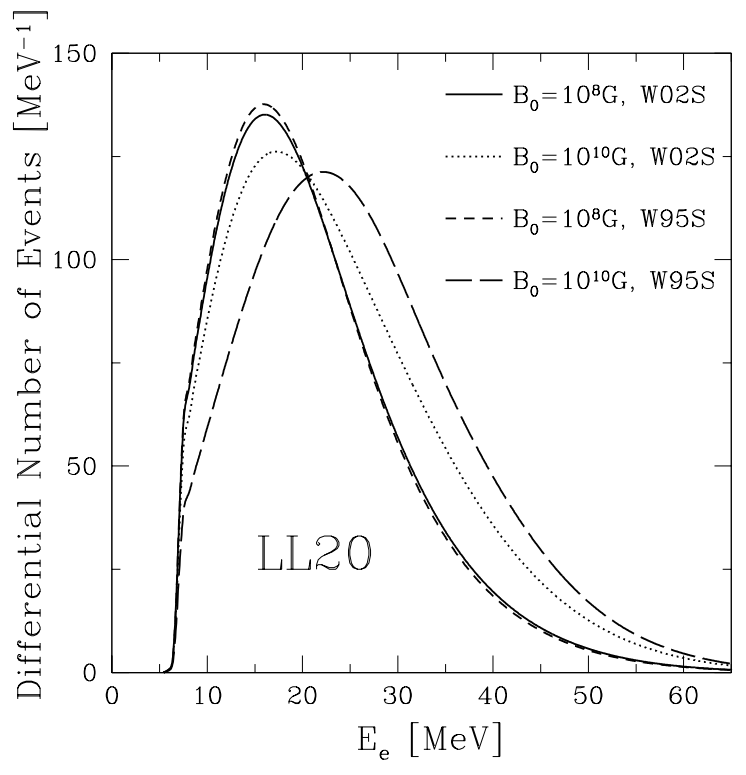

FIG. 6: Energy spectrum of electrons (positrons) at SK for the first $0.5 \mathrm{~s}$, obtained using the W02S and W95S presupernova models with $B_{0}=10^{8}, 10^{10} \mathrm{G}$. The original spectrum of LL20 is adopted.

G. Figure[7(a) is that for the LL20 model and Fig. 7(b) is for the TBP20 model; the shapes of the spectra from the other TBP11 and TBP15 models are almost the same as that with the TBP20 model, although the absolute value is different. When $B_{0}=10^{8} \mathrm{G}$, because the RSF conversions are absent and pure MSW flavor transitions occur, the energy spectra of the two models W02S and W02Z are degenerate. For the W02Z model with $B_{0}=10^{10}$ $\mathrm{G}$, since efficient flavor conversions occur at low energies but not in the high energy region, the event numbers are suppressed only in the low energy region and high energy tail is almost the same as that with $B_{0}=10^{8} \mathrm{G}$. Since the average energy difference between the $\bar{\nu}_{e}$ 's and $\nu_{\mu, \tau}$ 's is not prominent for the TBP models, the difference between energy spectra with various presupernova models is suppressed, compared with the LL20 model, particularly in the high energy tail.

\section{DISCUSSION}

\section{A. What can we learn about the RSF effect from the neutrino signal?}

In the previous section, it was shown that the RSF conversion strongly depends on the presupernova models of various metallicities. The behavior of the flavor mixing in the supernova envelope is very different from one presupernova model to another, and they indicate various profiles in supernovae such as magnetic field strength and the value of $\left(1-2 Y_{e}\right)$ at the resonance points as well as 


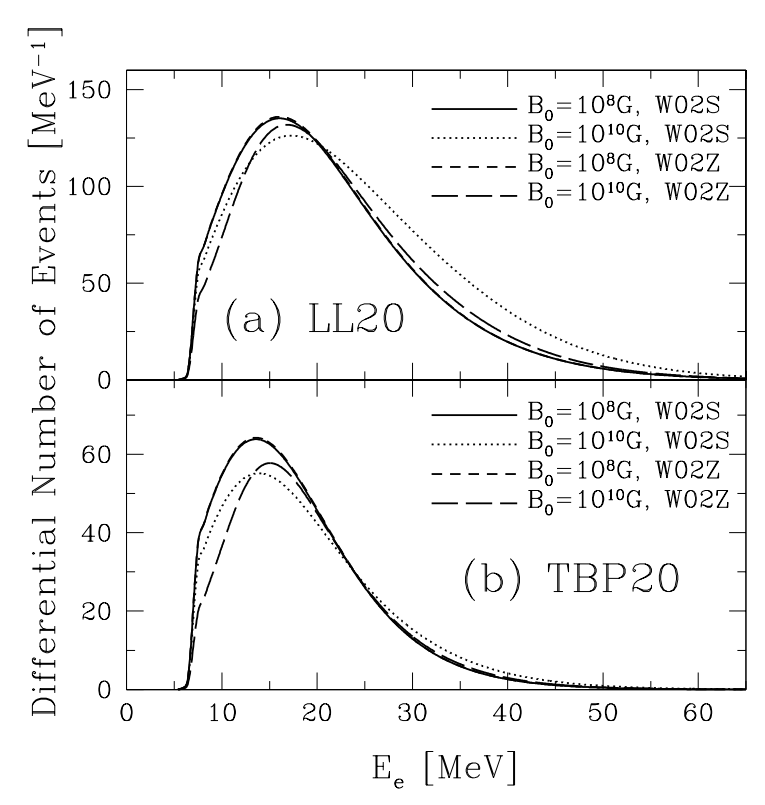

FIG. 7: Energy spectrum of electrons (positrons) at SK obtained using the W02S and W02Z presupernova models with $B_{0}=10^{8}, 10^{10}$ G. (a) The LL20 model for the first $0.5 \mathrm{~s}$. (b) The TBP20 model for the first $0.25 \mathrm{~s}$.

the neutrino magnetic moment. Unfortunately, however, what we can observe is the energy spectrum of electrons (positrons) at detectors on the Earth alone, and much information on the detailed character is lost, and therefore we can obtain only rather rough characteristics in principle. Here, we use as a simple indicator of the RSF conversions the following quantity:

$$
R_{\mathrm{SK}}=\frac{\text { number of events for } E_{e}>25 \mathrm{MeV}}{\text { number of events for } E_{e}<20 \mathrm{MeV}} .
$$

The values of $R_{\mathrm{SK}}$ for various models are summarized in Table \ Even if we use the data for the first 0.25 or 0.5 $\mathrm{s}$, it appears that statistically sufficient discussions are possible.

TABLE I: The values of $R_{\mathrm{SK}}$ for various models. Attached errors are statistical ones at the $1 \sigma$ level.

\begin{tabular}{lcccc}
\hline \hline & \multicolumn{2}{c}{$B_{0}=10^{8} \mathrm{G}$} & \multicolumn{2}{c}{$B_{0}=10^{10} \mathrm{G}$} \\
Model & W02S & W02Z & W02S & W02Z \\
\hline LL20 $(0.5 \mathrm{~s})$ & $0.59 \pm 0.02$ & $0.59 \pm 0.02$ & $0.96 \pm 0.04$ & $0.72 \pm 0.03$ \\
LL20 $(0.25 \mathrm{~s})$ & $0.41 \pm 0.03$ & $0.41 \pm 0.03$ & $0.71 \pm 0.04$ & $0.52 \pm 0.03$ \\
TBP20 & $0.25 \pm 0.02$ & $0.25 \pm 0.02$ & $0.35 \pm 0.03$ & $0.32 \pm 0.03$ \\
TBP15 & $0.21 \pm 0.03$ & $0.21 \pm 0.03$ & $0.33 \pm 0.04$ & $0.27 \pm 0.03$ \\
TBP11 & $0.22 \pm 0.03$ & $0.22 \pm 0.03$ & $0.35 \pm 0.03$ & $0.28 \pm 0.03$ \\
\hline \hline
\end{tabular}

In practice, to make matters worse, there is a large uncertainty concerning the original neutrino spectrum emitted by the core collapse; actually the values of $R_{\mathrm{SK}}$ are very different between the LL20 and TBP20 models (see Table 【). Thus, we must reduce the systematic errors of the models in discussing the RSF effect from the energy spectrum obtained. At present, however, this problem is very difficult and there is no way but to wait for the future development of numerical simulation of supernova explosions. Therefore, at present, it is very difficult to say even whether the RSF effect actually occurred or not.

If the systematic errors concerning the original neutrino spectrum are considerably reduced by future development of numerical simulations, it is expected that useful implications for the RSF mechanism will be obtained from the value of $R_{\mathrm{SK}}$. Therefore, at the end of this subsection, we discuss to what extent we can learn from the observed neutrino signal assuming that the systematic errors are much reduced. If the magnetic field or the neutrino magnetic moment are too small to induce adiabatic RSF conversions, the metallicity of presupernova stars is not detectable as shown in the second and third columns of Table【 On the other hand, in the case of a strong magnetic field like $B_{0}=10^{10} \mathrm{G}$, the value of $R_{\mathrm{SK}}$ becomes larger than that for $B_{0}=10^{8} \mathrm{G}$ at the $\gtrsim(2-3) \sigma$ level, which is a statistically meaningful number. However, it is very difficult to estimate the metallicity of the presupernova star, because the value of $R_{\mathrm{SK}}$ sensitively depends on the value of $B_{0}$, when it is larger than $10^{9} \mathrm{G}$ as shown in Ref. [26]. Consequently, even if the systematic errors were considerably reduced in the future, all we could say concerning the RSF effect from the galactic supernova neutrino burst would be at most that the RSF conversions have occurred or not. Other detailed discussion concerning various quantities such as the magnetic field strength, supernova metallicity, and neutrino magnetic moment would not be accessible.

\section{B. Future prospects}

In the near future, it is expected that the many uncertainties which complicate the observations of the RSF conversion will be much reduced. These uncertainties are, e.g., the neutrino magnetic moment, the supernova magnetic field structure, and the metallicity of the supernova.

If KamLAND receives a positive signal for the appearance of solar $\bar{\nu}_{e}$, then it indicates that the spin-flavor conversion of the Majorana neutrino actually occurs inside the Sun, and from the data we can obtain implications for the nonzero value of the neutrino magnetic moment. Actually, in the Sun the relevant RSF conversion is $\nu_{e} \leftrightarrow \bar{\nu}_{\mu, \tau}$, whereas in supernovae it is $\bar{\nu}_{e} \leftrightarrow \nu_{\mu, \tau}$. However, if the neutrinos are Majorana particles, the absolute values of the transition magnetic moments, which are responsible for the above two processes, are the same (the sign is different; $\mu_{i j}=-\mu_{j i}$ ). Thus, depending on the value that is observed by the solar neutrinos, it can easily be estimated whether the RSF effect is the relevant conversion process for the supernova neutrinos; further, 
if it is the relevant process, the magnetic field strength itself, not the combination $\mu_{\nu} B$, at the resonance points can be inferred. From future KamLAND results we can constrain only one of the magnetic moment tensor elements, which consist of three independent quantities, but we need all three values for the supernova case. Yet that information, if actually obtained, would be helpful for the estimation of other values and very useful.

As for the metallicity, we will obtain useful inferences from astrophysical discussions. The lifetime of massive stars which end their life by gravitational collapse is much shorter than that of the Sun, and the progenitors of observed supernovae are, therefore, younger. Consequently, the metallicity of the galactic supernovae is expected to be at least the solar abundance or more metal rich. If the metallicity is higher than that of the Sun, the suppression of $\left(1-2 Y_{e}\right)$ will be weaker and the RSF conversion will incline to be more adiabatic. On the other hand, the large and small Magellanic clouds are known to be very metal-poor systems [36, 37]. Thus, the determination of the explosion site will provide several rough estimations of the metallicity. Even though the explosion occurred in an optically thick environment such as the galactic center, the detected neutrinos alone could locate the supernova precisely; from the event number the distance to a supernova will be inferred, and from the event distribution the supernova direction can be determined [38].

Another object that is related to the metallicity effect is the supernova relic neutrino (SRN) 39, 40]. Because the SRN is the accumulation of neutrinos from all the past supernovae, the SRN includes neutrinos from supernovae with quite low metallicity in the early phase of galaxy formation. Recently, the SK collaboration released the first result of their search for the SRN signal [41], and it is only about a factor of 3 larger than the theoretical predictions by Ando et al. [39]. Data accumulation for several more years may be decisive for actual detection and setting a severe constraint on the SRN flux. However, because the totally time-integrated neutrino spectrum during the neutrino burst is needed for the SRN calculation, it is very difficulty to estimate the SRN flux including the RSF effect, which is affected by the time-dependent density and magnetic field profiles. Thus, it will be difficult to derive useful information about the RSF conversions from future SRN observations.

\section{CONCLUSION}

The RSF conversion of supernova neutrinos was investigated in detail; this conversion is induced by a nonzero magnetic moment of neutrinos and a strong supernova magnetic field. Because of the effective potential for the RSF conversion which delicately depends on the deviation of $Y_{e}$ from 0.5 , we studied the dependence of the RSF conversion on the adopted presupernova models. As the models, we used recent models by Woosley et al. 27] with solar and zero metallicities (W02S and W02Z) as well as that by Woosley and Weaver 28] with solar metallicity (W95S), which was the only model adopted in our previous paper [26], for comparison.

The W02 models were calculated using a recent shell model and resulted in substantial revisions to the older data sets in the W95S model. As a result, for both W02 models the value of $\left(1-2 Y_{e}\right)$ suddenly drops at the radius where the RSF conversions occur, leading to a less efficient RSF conversion than that with the W95S model, whose $\left(1-2 Y_{e}\right)$ gradually decreases at the RSF regions. In particular, for the $\mathrm{W} 02 \mathrm{Z}$ model, the value of $\left(1-2 Y_{e}\right)$ becomes exactly zero at the boundary between the $\mathrm{Si}+\mathrm{O}$ and $\mathrm{O}+\mathrm{Ne}+\mathrm{Mg}$ layers, which makes the conversion almost completely nonadiabatic. Thus, we found that energy spectra expected at SK for the W02 models would not be as hard as those for the W95S model. Unfortunately, however, there are many uncertainties at present, which are concerned with, e.g., the original neutrino spectrum, the neutrino magnetic moment, the supernova magnetic field structure, and the supernova metallicity. All these uncertainties complicate the discussions, and it is very difficult to obtain some useful information on the supernova metallicity or magnetic field strength, because the detected energy spectrum does not indicate which effect contributes to what extent.

In the future, however, it is expected that the development of numerical simulations of supernova explosions will much reduce the uncertainties for the original neutrino spectrum, and the KamLAND solar $\bar{\nu}_{e}$ observation, whether detected or not, will provide useful information on the neutrino magnetic moment. We believe that all these developments will enable a more decisive investigation of the RSF conversion of supernova neutrinos.

\section{Acknowledgments}

S. A.'s work is supported by Grants-in-Aid for JSPS Fellows. K. S.'s work is supported in part by Grantsin-Aid for scientific research provided by the Ministry of Education, Science and Culture of Japan through Research Grant No. S14102004 and No. S14079202.
[1] G. G. Raffelt, Nucl. Phys. B (Proc. Suppl.) 110, 254 (2002).
[2] A. S. Dighe and A. Y. Smirnov, Phys. Rev. D 62, 033007 (2000). 
[3] K. Takahashi and K. Sato, hep-ph/0205070.

[4] A. Cisneros, Astrophys. Sp. Sci. 10, 87 (1970).

[5] K. Fujikawa and R. E. Shrock, Phys. Rev. Lett. 45, 963 (1980).

[6] J. Schechter and J. W. F. Valle, Phys. Rev. D 24, 1883 (1981).

[7] J. Schechter and J. W. F. Valle, Phys. Rev. D 25, 283(E) (1982).

[8] C. S. Lim and W. J. Marciano, Phys. Rev. D 37, 1368 (1988).

[9] E. K. Akhmedov, Sov. J. Nucl. Phys. 48, 382 (1988).

[10] E. K. Akhmedov, Phys. Lett. B 213, 64 (1988).

[11] L. Wolfenstein, Phys. Rev. D 17, 2369 (1978).

[12] S. P. Mikheyev and A. Y. Smirnov, Sov. J. Nucl. Phys. 42, 913 (1985).

[13] S. P. Mikheyev and A. Y. Smirnov, Nuovo Climento C 9, 17 (1986).

[14] J. Barranco, O. Miranda, T. Rashba, V. Semikoz, and J. Valle, Phys. Rev. D 66, 093009 (2002).

[15] K. Eguchi et al., Phys. Rev. Lett. 90, 021802 (2003).

[16] E. Torrente-Lujan, hep-ph/0302082.

[17] A. Ayala, J. C. D'Olivo, and M. Torres, Phys. Rev. D 59, 111901 (1999).

[18] M. B. Voloshin, Phys. Lett. B 209, 360 (1988).

[19] E. K. Akhmedov and Z. G. Berezhiani, Nucl. Phys. B 373, 479 (1992).

[20] E. K. Akhmedov, S. T. Petcov, and A. Y. Smirnov, Phys. Rev. D 48, 2167 (1993).

[21] J. T. Peltoniemi, Astron. Astrophys. 254, 121 (1992).

[22] H. Athar, J. T. Peltoniemi, and A. Y. Smirnov, Phys. Rev. D 51, 6647 (1995).

[23] T. Totani and K. Sato, Phys. Rev. D 54, 5975 (1996).
[24] H. Nunokawa, Y. Z. Qian, and G. M. Fuller, Phys. Rev. D 55, 3265 (1997).

[25] H. Nunokawa, R. Tomàs, and J. W. F. Valle, Astropart. Phys. 11, 317 (1999).

[26] S. Ando and K. Sato, Phys. Rev. D 67, 023004 (2003).

[27] S. E. Woosley, A. Heger, and T. A. Weaver, Rev. Mod. Phys. 74, 1015 (2002).

[28] S. E. Woosley and T. A. Weaver, Astrophys. J. Suppl. Ser. 101, 181 (1995).

[29] A. Heger, S. E. Woosley, G. Martínez-Pinedo, and K. Lnaganke, Astrophys. J. 560, 307 (2001).

[30] R. C. Schirato and G. M. Fuller, astro-ph/0205390.

[31] K. Takahashi, K. Sato, H. E. Dalhed, and J. R. Wilson, astro-ph/0212195.

[32] G. Ghanmugam, Ann. Rev. Astron. Astrophys. 411, 823 (1992).

[33] T. Totani, K. Sato, H. E. Dalhed, and J. R. Wilson, Astrophys. J. 496, 216 (1998).

[34] T. A. Thompson, A. Burrows, and P. Pinto, astro$\mathrm{ph} / 0211194$.

[35] A. Strumia and F. Vissani, astro-ph/0302055; P. Vogel and J. F. Beacom, Phys. Rev. D 60, 053003 (1999).

[36] M. Hilker, T. Richtler, and W. Gieren, Astron. Astrophys. 294, 648 (1995).

[37] M. T. Meliani, B. Barbuy, and T. Richtler, Astron. Astrophys. 304, 347 (1995).

[38] S. Ando and K. Sato, Prog. Theor. Phys. 107, 957 (2002).

[39] S. Ando, K. Sato, and T. Totani, Astropart. Phys. 18, 307 (2003).

[40] S. Ando and K. Sato, Phys. Lett. B 559, 113 (2003).

[41] M. Malek et al. (The Super-Kamiokande Collaboration), Phys. Rev. Lett. 90, 061101 (2003). 